\title{
Moving the eyes along the mental number line: Comparing SNARC effects with saccadic and manual responses
}

\author{
WOLF SCHWARZ and INGE M. KEUS \\ University of Nijmegen, Nijmegen, The Netherlands
}

\begin{abstract}
Bimanual parity judgments about numerically small (large) digits are faster with the left (right) hand, even though parity is unrelated to numerical magnitude per se (the SNARC effect; Dehaene, Bossini, \& Giraux, 1993). According to one model, this effect reflects a space-related representation of numerical magnitudes (mental number line) with a genuine left-to-right orientation. Alternatively, it may simply reflect an overlearned motor association between numbers and manual responses-as, for example, on typewriters or computer keyboards-in which case it should be weaker or absent with effectors whose horizontal response component is less systematically associated with individual numbers. Two experiments involving comparisons of saccadic and manual parity judgment tasks clearly support the first view; they also establish a vertical SNARC effect, suggesting that our magnitude representation resembles a number map, rather than a number line.
\end{abstract}

Daily life constantly confronts us with numerical information that we use to better apprehend and describe our environment and to plan and guide actions that we take. For example, information about many important parameters of our environment, such as distances, dates, temperatures, weights, ages, prices, or exam grades, are usually communicated in formats involving numbers. Accordingly, the characteristics of how we represent numerical information have been the subject of considerable research over the last 2 decades that has led to a detailed understanding of how we process numerical information, of how these cognitive skills develop from early age on, and also of the neuronal circuits underlying the various forms of numerical processing in humans (for detailed summaries, see Ashcraft, 1992; Besner \& Coltheart, 1979; Butterworth, 1999; Corballis, 1994; Dehaene, 1997; Dehaene, Dehaene-Lambertz, \& Cohen, 1998; Gallistel \& Gelman, 1992; McCloskey, 1992).

An influential concept that has emerged from this work is the translation of digits into percept-like analogue representations, a continuous or fine-graded more-or-less code, much like sensory representations of extensive physical attributes such as color or contrast. This analogue magnitude representation is thought to be fast, inaccurate, and preverbal; it is assumed to be a prerequisite developmental stage to the build-up of slower, but exact, verbal algorithms that form the basis of our abstract (e.g., algebraic) computational cognitive skills. Evidence sup-

We thank Gerard van Oijen for his excellent technical assistance. Correspondence concerning this article should be addressed to W. Schwarz, University of Potsdam, Department of Psychology, P. O. Box 6015 53, D-14415 Potsdam, Germany (e-mail: wschwarz@rz.unipotsdam.de). porting these conclusions comes from experimental, comparative, physiological, and developmental psychology and has recently been extensively reviewed (Butterworth, 1999; Dehaene, 1997; Dehaene et al., 1998). The analogue mode of numerical processing is thought to be based on a distributed representation, not unlike the sensory representation of extensive object features. More specifically, numbers are often assumed to be represented along a mental number line (Restle, 1970), and various detailed, process-oriented models have been proposed to explain how different magnitudes are coded as space-related representations along this hypothetical number line (e.g., Dehaene, 1997; Gallistel, 2002; Schwarz \& Ischebeck, 2000; Schwarz \& Stein, 1998).

\section{The SNARC Effect as Evidence for the Mental Number Line}

Just how literally can this metaphor of a mental number line be taken? For example, if numbers are internally represented along a mental line, what is this line's direction of orientation? One line of evidence addressing these questions comes from brain-imaging studies indicating a significant overlap of the brain areas activated by numerical tasks and by tasks requiring the processing of spatial or location information (Chochon, Cohen, van de Moortele, \& Dehaene, 1999; Dehaene et al., 1998; Dehaene, Spelke, Pinel, Stanescu, \& Tsivkin, 1999; Göbel, Walsh, \& Rushworth, 2001). More specifically, numerical tasks activate regions in the inferior parietal cortex, a brain region that is well known to be actively involved in the coding of spatial representations as well (e.g., Colby \& Goldberg, 1999). Supporting this view, Zorzi, Priftis, and Umiltà (2002) have reported that patients with a neglect of the left visual field due to a right parietal lesion 
exhibit systematic rightward shifts in a number bisection task - as if they neglected the left portion of the mental number line-whereas control patients and healthy normals have no systematic problems with this task. Conceivably, then, our number representation may be inherently space related because it exploits or coactivates neural networks that deal primarily with the processing of spatial information.

Further important evidence regarding the abovementioned questions is provided by a study of Dehaene, Bossini, and Giraux (1993), who asked participants to indicate the parity (odd vs. even) of digits with bimanual (left- vs. right-hand) responses. They found that response times (RTs) were systematically modulated by the digit's magnitude: Even though numerical magnitude per se was, in principle, irrelevant for judging the digit's parity, small digits (e.g., 1,2) were responded to more quickly with the left than with the right hand, whereas large digits (e.g., 8, 9) were responded to more quickly with the right than with the left hand. This systematic spatialnumerical association of response code (SNARC effect) was observed across a considerable range of different experimental conditions involving variations of the participant's handedness and the number's notational format. Note that the SNARC effect is independent of the main effects of left versus right hand and odd versus even responses; rather, it refers to the interaction of the responding hand (left vs. right) with numerical magnitude. Subsequent research has confirmed the basic SNARC effect, studied its developmental changes from young age on (Berch, Foley, Hill, \& Ryan, 1999), and extended it to less number-related tasks, such as phonemic monitoring (Fias, 2001; Fias, Brysbaert, Geypens, \& d'Ydewalle, 1996). According to Dehaene (1997; Dehaene et al., 1993; for related views, see Bächtold, Baumüller, \& Brugger, 1998; Butterworth, 1999; Fias, 2001; Fias et al., 1996; Fischer, 2001; Fischer, Castel, Dodd, \& Pratt, 2003), these findings collectively suggest two basic interrelated conclusions. The first conclusion is that under some conditions, presenting a number seems to call up an internal magnitude representation - even in situations in which magnitude per se is logically irrelevant and could, therefore, be in principle ignored (cf. Otten, Sudevan, Logan, \& Coles, 1996; Schwarz \& Heinze, 1998; Sudevan \& Taylor, 1987). This conclusion fits in with related Stroop-type congruency effects obtained in interference experiments in which humans judge the physical size of digits or whether two digits are physically the same or different. In these tasks, the digit's numerical magnitude is also, in principle, irrelevant but still systematically modulates the observed response latencies (Algom, Dekel, \& Pansky, 1996; Dehaene \& Akhavein, 1995; Girelli, Lucangeli, \& Butterworth, 2000; Pansky \& Algom, 1999; Schwarz \& Ischebeck, 2003; Tzelgov, Meyer, \& Henik, 1992).

Second, Dehaene et al.'s (1993) findings also suggest the notion that this magnitude representation is inherently space related and, therefore, amenable to spatial- numerical association effects. More specifically, the internal representation of numbers seems to be such that increasing numerical magnitudes are ordered in a leftto-right orientation along a hypothetical mental number line (e.g., Restle, 1970).

\section{The Manual Association Hypothesis: Eye and Hand Dissociated?}

Although the first conclusion seems to be widely accepted for many conditions and tasks (but see Pansky \& Algom, 1999, 2002, for a systematic and careful exploration of the boundary conditions), alternative views regarding the second conclusion are conceivable (see, e.g., Bächtold et al., 1998). For example, there is a growing body of evidence showing that many effects previously thought to be purely cognitive in nature are, to a surprising degree, effector specific; for example, Milner and Goodale (1995) have demonstrated how manual and visual grasping responses can be systematically dissociated. More recently, Glover and Dixon $(2001,2002)$ have advanced a general model in which the perceptually guided planning of motor actions, such as reaching or grasping, and the on-line-controlled actual execution of this action are systematically dissociated. In support of their proposal, they demonstrated that in a grasping task, contextinduced illusions systematically distorted the perception of the target disk size and the initial planning of a motor action, whereas the actual execution of the motor action, as measured by grip aperture, revealed a steadily decreasing effect of the illusion (on-line action control). On this general view, the SNARC effect might not so much reflect the nature of our internal representation of numerical magnitude per se (perception) but, rather, result from a highly overlearned motor association between particular numbers and particular manual responses (action) - largely independently of how these numbers are internally represented. To illustrate (and overstate) this argument with a simplified analogy, consider that most Europeans are used to associate, and to handle more efficiently, forks and knives with the left and the right hands, respectively. However, it seems unlikely that this fork-left and knife-right superiority (as compared with the converse mapping) results from a space-related internal representation of cutlery. Rather, it simply reflects the fact that particular motor effectors are strongly associated and, thus, more effective with particular objects. For example, Bächtold et al. found that when participants had to relate numbers to locations on a ruler, the usual SNARC effect was replicated; however, when the same numbers had to be related to the circular face of a standard analogue clock (i.e., smaller numbers right, larger numbers left), a reverse SNARC effect was obtained. Other obvious examples in the context of number processing are slide rulers, typewriters, and computer keyboards - instruments in which small versus large numbers are regularly associated with the activation of the left versus the right hand, respectively. For example, professional typists are trained to use a digit-to-hand map- 
ping such that the left hand covers the digits $1-5$ and the right hand the digits 6-9, and even less professional users of standard keyboards apply essentially the same digitto-hand mapping. According to this alternative manual association hypothesis, the SNARC effect would thus not so much reflect an internal, genuinely space-related number representation but, rather, result from the fact that we have available highly overlearned (and thus very efficient) number-to-hand motor associations. In contrast, according to the number line interpretation of the SNARC effect, the advantage of the left (right) hand with small (large) numbers is just a consequence of our inherently space-related mental representation of magnitude.

Part of the existing evidence seems clearly more in line with the mental number line interpretation of the SNARC effect. For example, Dehaene et al. (1993, Experiment 6) observed a normal SNARC effect in a condition in which the participants crossed their hands to push the left (right) button with the right (left) hand. The manual association hypothesis can account for this finding only with the additional assumption that the critical association is not necessarily with the specific hand but, within a more abstract body scheme, with that manual effector that currently is placed on the respective side in extracorporal space (see Latash, 1999; Rosenbaum, 1991; Schmidt, 1988). Some open questions related to the interpretation of the crossed-hands condition remain, however (for a recent critical discussion of crossed-hands experiments, see Wascher, Schatz, Kuder, \& Verleger, 2001). For example, if the SNARC effect does indeed depend exclusively on the extracorporal spatial reference, one would be led to expect that only the horizontal spatial separation of the two response buttons could set the spatial frame of reference, and not the placement (be it crossed or uncrossed) of the actual effectors. One should then, for example, expect the SNARC effect to decrease with decreasing button separation and, eventually, to vanish with response buttons placed right above each other. However, contrary to this expectation, the effect is virtually identical over a more than tenfold range of horizontal separations from $25 \mathrm{~mm}$ (Fias et al., 1996) to $260 \mathrm{~mm}$ (Dehaene et al., 1993). Clearly, this finding seems to be difficult to reconcile with the notion that the SNARC effect directly reflects the (in-)congruency of internal number representations and external response requirements and invites alternative accounts, such as the manual association hypothesis.

Further relevant evidence comes from experiments showing that the SNARC effect depends on the current numerical context (Dehaene et al., 1993, Experiment 3; Fias et al., 1996, Experiment 1). That is, there are separate SNARC effects within each of the subintervals $[0,5]$ and $[4,9]$ when these are presented in different experimental blocks. Specifically, the digits 4 and 5 are responded to more quickly with the right than with the left hand when the interval is $[0,5]$ but more quickly with the left than with the right hand when the interval is $[4$, 9]. Again, for the manual association hypothesis, this finding requires that the motor associations assumed by this hypothesis cannot be thought of as immutable hardwired bonds; rather, they need to be reprogramable depending on the current numerical context (cf. Rosenbaum, 1991). In this respect, the manual association hypothesis stands perhaps on a par with the number line interpretation, because even with an internal number line representation (at least in its strict interpretation; see, e.g., Restle, 1970), it is not immediately obvious why, for example, the digit 5 is sometimes represented as small (left) and sometimes as large (right). That is, both rivaling hypotheses need to assume some degree of flexibility to explain the adaptation to different numerical contexts.

How, then, could the manual association hypothesis be tested in a more stringent, direct way and be contrasted with predictions based on the number line interpretation of the SNARC effect? Evidently, the manual association hypothesis makes one direct, strong prediction - namely, that the SNARC effect should be weaker or absent in response tasks with effectors whose horizontal response amplitude is not (or is less) systematically associated with small versus large numbers. For example, under the manual association hypothesis, we would not expect to obtain a SNARC effect with left versus right saccadic responses, because eye movements to the left versus the right are less systematically associated with small versus large numbers than are manual responses. In terms of the above-mentioned simplified analogy, although for many Europeans the left (right) hand more efficiently grabs and handles forks (knives), one would certainly not expect a corresponding superiority to hold for saccadic responses toward forks versus knives as well. A very similar argument holds for numbers: For example, in typing in a number on a computer keyboard, we manually always associate digits such as 1 and 2 with left and digits such as 8 and 9 with right, even if the to-be-produced compound number (e.g., 8921) has large leading (i.e., left) digits and small trailing (i.e., right) digits. However, unlike its manual production, the sequential visual readout of the produced number is, in general, not systematically related to the digit's magnitudes; for example, in reading the centrally presented number 8921 , the larger digits require a saccade to the left, the smaller digits a saccade to the right. In this sense, then, our hands (e.g., in typing in digits) are more systematically related to small versus large numbers than are our eyes (e.g., in the visual readout of written numbers). If, on the other hand, the SNARC effect indeed reflects an inherently spacerelated mental magnitude representation, it should presumably occur in a similar way with any effector that is capable of distinct left versus right responses.

Unfortunately, all inquiries into the SNARC effect so far have exclusively relied on comparing left versus right manual responses (for a related but different paradigm, see Fischer, 2001), so that, from extant data, it is very difficult to systematically evaluate these different accounts. To overcome this problem, in the present exper- 
iments, we studied the SNARC effect by using saccadic response conditions in which the participants indicated the parity of a digit by making eye movements to the left or the right. As in the standard bimanual task, the mapping of parity to response side was varied so that, within each digit, the latencies of saccades to the left and to the right could be compared. If the number line interpretation of the SNARC effect holds, we would expect that the difference between onset latencies of saccades to the right minus onset latencies of saccades to the left should decrease with numerical magnitude. In contrast, the manual association hypothesis predicts an eye-hand dissociation (cf. Glover \& Dixon, 2001, 2002; Milner \& Goodale, 1995) for parity judgments, so that the difference in right minus left saccadic onset latency (if there is any) should be essentially independent of numerical magnitude.

\section{Is There a Vertical SNARC Effect?}

In previous research, interpretations of the SNARC effect have centered around a hypothetical left-to-right orientation of our number representation, in the sense of a horizontal mental number line. As was discussed above, numerical tasks activate areas in the inferior parietal cortex, a structure that is known to be essential for the processing of spatial information. Given that our spatial representations are not limited to a single horizontal direction (cf. Rubin, Nakayama, \& Shapley, 1996), it is conceivable that our mental number representation uses spatial codes that are richer than strictly unidimensional formats. For example, Dehaene (1997, pp. 82-83) took into consideration the possibility that small numbers might also be internally represented as bottom and large numbers as top. Consistent with this conjecture, many languages use spatial metaphors, such as low and high, to denote small and large numbers, respectively. If Dehaene's conjecture is correct, strictly unidimensional concepts such as a number line might be incomplete descriptions of how we represent numerical magnitude and should be replaced with higher dimensional concepts, such as an internal number map or a number field. Indeed, many technical instruments, such as analogue thermometers or hand-held pocket calculators, are manufactured so that small numbers are associated with lower and larger numbers with upper locations. On the other hand, the key pads of telephones (small numbers at the top), conventional computer raster graphics (zero is the top display line), and rankings or league charts (number one listed at the top) are common exceptions to this rule.

Does, then, a genuine SNARC effect exist with respect to the vertical orientation as well? Given the left-right dichotomy inherent to our hands, this question would seem difficult to address using manual responses, because upper versus lower response locations are not as systematically related to either hand as are left versus right response buttons. On the other hand, if we could establish that eye movements do exhibit SNARC-like effects, the saccadic response condition described above would also allow us to further explore the internal topology of our numerical magnitude representations for nonhorizontal orientations as well. More specifically, comparing the latencies of upward versus downward saccades in a parity judgment task would then provide a direct and convenient way to test for potential processing advantages of small numbers with lower and of large numbers with upper response locations. Such a finding could be interpreted as evidence for a genuinely two-dimensional internal number representation, such as a number field or a number map. Alternatively, it would at least support the notion that there are two functionally independent number representations, or perhaps a single representation that has an adaptive, task-dependent spatial orientation.

In summary, the present study had three aims. First, we studied the SNARC effect under response conditions in which the participants had to indicate the parity of a digit with a left versus a right saccade. The manual association hypothesis predicts that under these conditions, no or, at best, a minuscule SNARC effect should be observed, whereas the number line interpretation does predict the standard effect. A second aim was to compare the size (if any) of the SNARC effect on latencies and errors under the saccadic response condition with the corresponding effects obtained under the standard bimanual response condition. Specifically, if the SNARC effect on latencies (manual or saccadic) indeed arises entirely during early representational processing stages preceding the activation of the required motor response, it should be of similar size for both the manual and the saccadic response conditions. Finally, to further explore the topology of our internal magnitude representations, we studied the SNARC effect under a saccadic response condition with vertical (i.e., upward vs. downward) saccades, to see whether a SNARC effect would arise under these conditions as well.

\section{EXPERIMENT 1}

The purpose of Experiment 1 was to test for and to compare the SNARC effect under two different response conditions: manual versus saccadic.

\section{Method}

\section{Participants}

Sixteen (13 female) right-handed students at the University of Nijmegen, 18-26 years of age, participated in two separate sessions in return for 25 Euro $(\approx \$ 25)$.

\section{Stimuli and Apparatus}

The stimuli consisted of the digits 0-9, which were presented in green using the TrueType Verdana font. Each digit was displayed in the center of a $100-\mathrm{Hz}, 480 \times 640$ pixel VGA color monitor against a dark background; the display timing was synchronized with the video refresh cycle. When viewed from a distance of $70 \mathrm{~cm}$, the digits subtended angles of about $2.0^{\circ} \times 1.2^{\circ}$. Manual response latencies were recorded to the nearest millisecond, using an external response keyboard attached to the computer's parallel port. The SMI EyeLink-Hispeed 2D system (SensoMotoric Instruments) was used to track both eyes, using a sampling rate of $250 \mathrm{~Hz}$; the spatial accuracy of this eye tracker is better than $0.01^{\circ}$, according to the man- 
ufacturer. To detect the onsets and offsets of saccades, three thresholds were used: a motion threshold of $0.1^{\circ}$, a velocity threshold of $30 \% \mathrm{sec}$, and an acceleration threshold of $8,000 \% / \mathrm{sec}^{2}$. To discourage the participants from making head movements, a chinrest was used throughout the experiment.

\section{Procedure}

The experiment consisted of two sessions on separate days, one for each mapping of parity to response side (even-left/odd-right vs. even-right/odd-left). In each session, the participants worked under both a manual and a saccadic response condition with the same parity-to-side mapping; the order of the response conditions within a session and of the mapping across sessions were counterbalanced across participants. In both response conditions, the task was to judge the parity of the number presented; the participants were instructed to respond as quickly as possible but to avoid errors. Between the two response conditions, there was a break of about $15 \mathrm{~min}$. Each complete session took approximately $2.5 \mathrm{~h}$.

Manual response condition. In the manual response condition with the even-left mapping, the participants were instructed to press the left button when the number was even and the right button when the number was odd. In the even-right condition, the mapping of parity to response side was reversed.

Each trial started with the presentation of a green fixation cross for $500 \mathrm{msec}$, which was then replaced with a single digit. Responses were given with the index fingers of the left and right hands; they terminated the stimulus display. The next trial started 1,200-1,400 msec (uniform distribution) later. RT was defined as the time from the onset of the digit to the onset of the buttonpress. No feedback was given for correct responses, whereas for incorrect responses the word "Error" was presented for $2 \mathrm{sec}$.

A single block contained 4 warm-up trials, followed by 50 regular trials, which were generated by the computer in a random order, subject to the boundary condition that within 2 consecutive blocks each digit preceded every other digit exactly once. Blocks were separated by breaks that could be terminated by the participant after a minimum duration of $20 \mathrm{sec}$. During the break, the number of remaining blocks, the number of errors in the last block, and the mean RT of the last block and of the fastest block so far were displayed on the screen. When the number of errors per block exceeded three, an extra feedback message asked the participant to be more accurate. The complete manual response condition of a session consisted of 12 blocks.

Saccadic response condition. In the saccadic response condition, white square boxes $(8 \times 8 \mathrm{~mm})$, with a small dot in the center of each, were presented continuously $8^{\circ}$ to the left and $8^{\circ}$ to the right of a central fixation cross. In the even-left mapping, the participants were instructed to saccade to the center dot in the left box when the number was even and to the center dot in the right box when the number was odd. In the even-right mapping condition, the mapping of parity to response side was reversed. After the participant had read the instructions and, if necessary, additional questions had been answered, a band carrying two micro video cameras was mounted to the participant's head, and the eye-tracking system was calibrated and validated.

Each trial in the saccadic response condition started with the presentation of a central green fixation cross for $500 \mathrm{msec}$, on which the participant had to fix his or her gaze; it was then replaced by a central digit that lasted for 2,200 msec. Saccadic latency (SL) was defined as the time from the onset of the digit to the onset of a saccade toward the center of one of the two boxes. A red cross was presented for 2,800-3,000 msec (uniform distribution) until the next trial started. During the entire trial, the $(x, y)$ coordinates of both eyes were stored on disk every $4 \mathrm{msec}$, together with a record of the time-stamped stimulus events on screen; these data were then analyzed off line.

Blocks were made up of 4 practice trials plus 50 regular trials and were separated by breaks, exactly as in the manual response condition, except that after each block, the participant received feedback only about the number of blocks completed and the number of blocks still remaining. The complete saccadic response condition of a session consisted of eight blocks.

\section{Preliminary Data Reduction}

Across blocks, mean manual RT decreased as an approximately exponential function that leveled off after about 2 blocks. Therefore, error rates and the mean RTs of the correct responses per condition were based on the final 10 blocks of the manual response condition. Trials with RTs shorter than $200 \mathrm{msec}$ or longer than $1,200 \mathrm{msec}$ (a total of $0.6 \%$ ) were excluded from all analyses.

For a trial from the saccadic response condition to be accepted as valid, it had to meet the following five criteria: (1) The preceding green central cross was properly fixated during the last $200 \mathrm{msec}$ before the digit appeared, (2) the saccade started between 200 and 1,200 msec after digit onset, (3) the horizontal saccade amplitude was between $5^{\circ}$ and $11^{\circ}$, (4) the vertical saccade amplitude was smaller than $3^{\circ}$, and (5) the saccade duration was between 20 and $120 \mathrm{msec}$. Across all participants, $25 \%$ of the trials did not meet one or more of these criteria; these invalid trials (most of them containing rapid microsaccades, improper initial fixations, or saccades not landing close enough to one of the two response boxes) were excluded from all further analyses. Perhaps due to the detailed calibration and validation procedure, SLs did not decrease consistently across blocks, so that error rates and mean correct SLs were based on all valid trials from the eight blocks.

Mean RTs and mean SLs were subjected to separate repeated measures $2 \times 2 \times 5$ analyses of variance (ANOVAs), with factors of parity ( 2 : even vs. odd), response side ( 2 : left vs. right), and numerical magnitude (classified into five bins: 0/1, 2/3, 4/5, 6/7, and $8 / 9$ ). As in Dehaene et al. (1993), we reduced the 10 digits into five bins of increasing magnitude, because in this way, within any given bin, RTs and SLs for left-side responses and right-side responses were based on responses to both digits of this bin and from both sessions; thus, any main effect of mapping (confounded with session) and of the individual digits should cancel out. For example, left-hand RTs for the largest bin (8/9) were based on responses to the digit 8 with the even-left mapping (one session) and on responses to the digit 9 with the odd-left mapping (the other session). Also, subsuming the digits into successive magnitude bins allowed for the orthogonal variation of magnitude and parity, which was impossible on the basis of individual digits.

Comparisons across the two response conditions were based on four-way repeated measures ANOVAs, with the additional factor of effector (2: hand vs. eye).

To statistically evaluate effects on error rates, we first applied the $\arcsin (\sqrt{ } p)$ transformation to the error rates (e.g., Bishop, Fienberg, $\&$ Holland, 1975, pp. 367ff), to achieve approximate variance equality, and then submitted these transformed values to ANOVAs of the same format as for RT and SL.

Unless noted otherwise, significant results reached at least the .05 level.

\section{Results}

\section{Manual Responses}

Response times. Overall, the participants responded more quickly with their right $(438 \mathrm{msec})$ than with their left $(460 \mathrm{msec})$ hands $\left[F(1,15)=43.61, M S_{\mathrm{e}}=890.44\right.$, $p<.001]$. Also, the two extreme magnitude bins $(0 / 1$ and $8 / 9)$ yielded longer RTs than did the three intermediate magnitude bins $\left[F(4,60)=5.84, M S_{\mathrm{e}}=418.88, p<.001\right]$. Parity had no main effect on RT $\left[F(1,15)=0.53, M S_{\mathrm{e}}=\right.$ $881.11, p>.45]$ but interacted with magnitude $[F(4,60)=$ 
6.06, $\left.M S_{\mathrm{e}}=4,052.79, p<.001\right]$-mainly because for both response mappings, in the fourth bin the digit 7 was classified as odd much more quickly $(434 \mathrm{msec})$ than the digit 6 was classified as even ( $458 \mathrm{msec})$, whereas for all other magnitude bins, odd and even judgments took very nearly the same time. Of most importance to our purposes, there was a strong interaction between response side and magnitude $\left[F(4,60)=9.95, M S_{\mathrm{e}}=479.12, p<\right.$ .001]. As is illustrated in Figure 1A, the RT difference between right-hand responses minus left-hand responses decreased monotonically with numerical magnitude; the pattern forms a funnel interaction because right-hand responses were generally faster than left-hand responses.
This SNARC effect occurs in a similar way for odd and even digits separately, so that the triple interaction of response side, magnitude, and parity was not significant $\left[F(4,60)=2.47, M S_{\mathrm{e}}=164.55, p>.05\right]$.

To further quantify the size of this manual SNARC effect, we first regressed, for each participant individually, the difference of mean right-hand RT minus mean lefthand RT on the 10 values of numerical magnitude (0-9; cf. Dehaene et al., 1993; Fias et al., 1996). The obtained regression slope values were then averaged across participants and evaluated by $t$ tests. The mean slope was equal to $-5.8[t(15)=-4.54, S E M=1.28, p<.001]$, meaning that, on average, the difference between right-
A

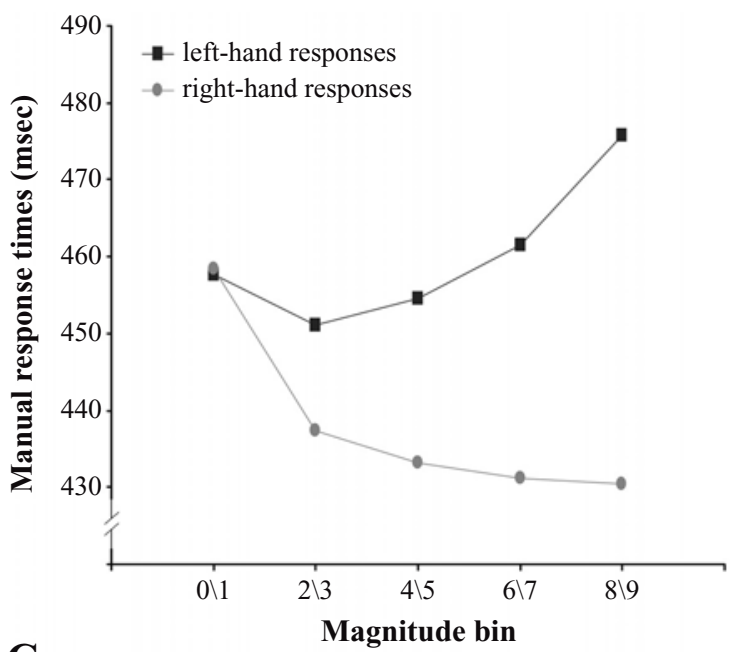

C

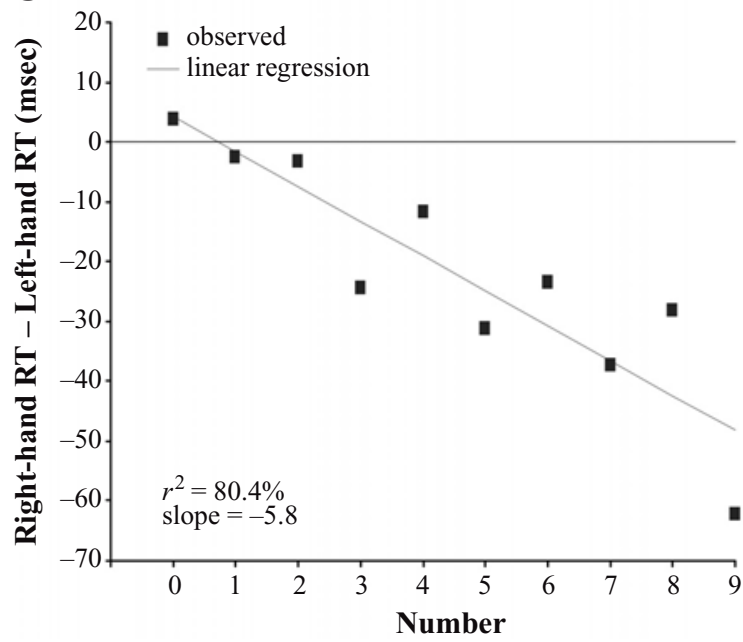

B

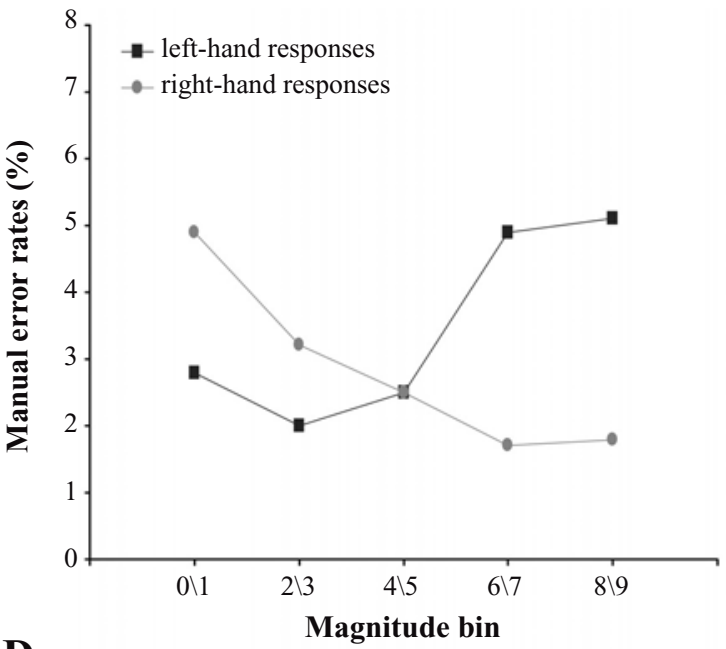

D

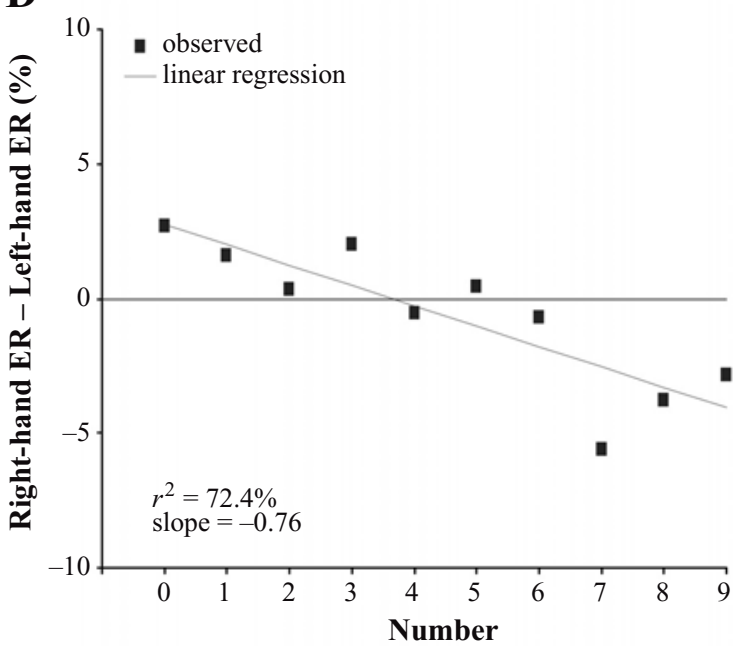

Figure 1. (A) Mean response times (in milliseconds) in the manual response condition in Experiment 1 for left-hand responses (squares) and right-hand responses (dots) as a function of numerical magnitude. (B) Error rates (in percentages) in the manual response condition in Experiment 1 for left-hand responses (squares) and right-hand responses (dots) as a function of numerical magnitude. (C) Difference in mean response times (RTs, in milliseconds) between right-hand responses and left-hand responses as a function of number $(0,1,2, \ldots, 9)$ in the manual response condition in Experiment 1. Solid line: regression of RT difference on number. (D) Difference in error rates (ERs, in percentages) between right-hand responses and left-hand responses as a function of number $(0,1,2, \ldots, 9)$ in the manual response condition in Experiment 1 . Solid line: regression of ER difference on number. 
hand versus left-hand RTs decreased by $5.8 \mathrm{msec}$ per digit.

Error rates. The overall error rate was 3.1\%, and only two effects modulated it significantly. First, a clear SNARC effect (shown in Figure 1B) on error rates was obtained: With increasing numerical magnitude, error rates decreased for right-hand responses but increased for left-hand responses $\left[F(4,60)=8.11, M S_{\mathrm{e}}=0.014\right.$, $p<.001]$. Second, this SNARC effect on error rates differed slightly for odd and even digits, resulting in a significant three-way interaction of response side, magnitude, and parity $\left[F(4,60)=4.91, M S_{\mathrm{e}}=0.006, p<.02\right]$.

\section{Saccadic Responses}

Saccadic latency. Overall, the participants saccaded faster to the right $(397 \mathrm{msec})$ than to the left $(408 \mathrm{msec})$ $\left[F(1,15)=4.91, M S_{\mathrm{e}}=2,268.25, p<.05\right]$. Neither numerical magnitude nor parity exerted a main effect on SL. Only two interaction effects on SL were significant. First, parity interacted with magnitude $[F(4,60)=5.20$, $\left.M S_{\mathrm{e}}=1,183.96, p<.005\right]$ - again, mainly because, for both response sides, the digit 7 was classified as odd much more quickly $(381 \mathrm{msec})$ than the digit 6 was classified as even $(421 \mathrm{msec})$, whereas for all other magnitude bins, odd and even judgments took nearly the same
A
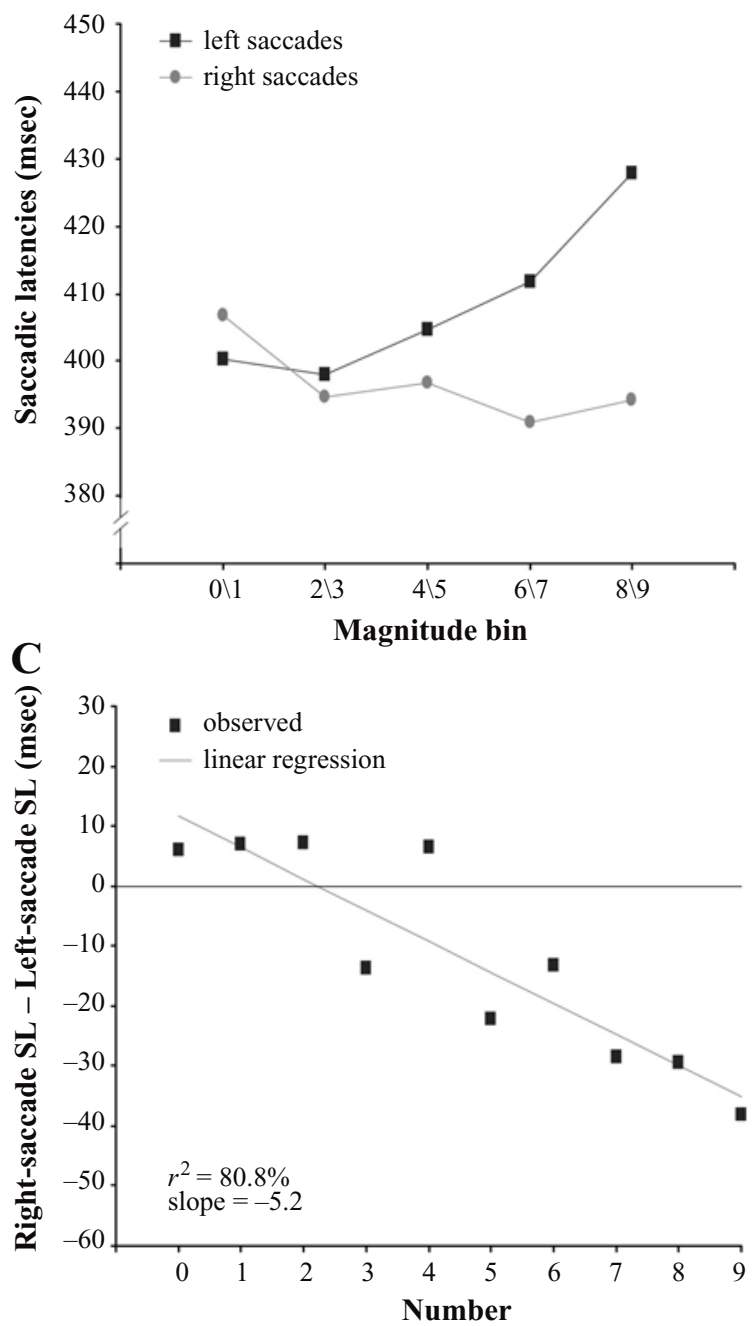

B
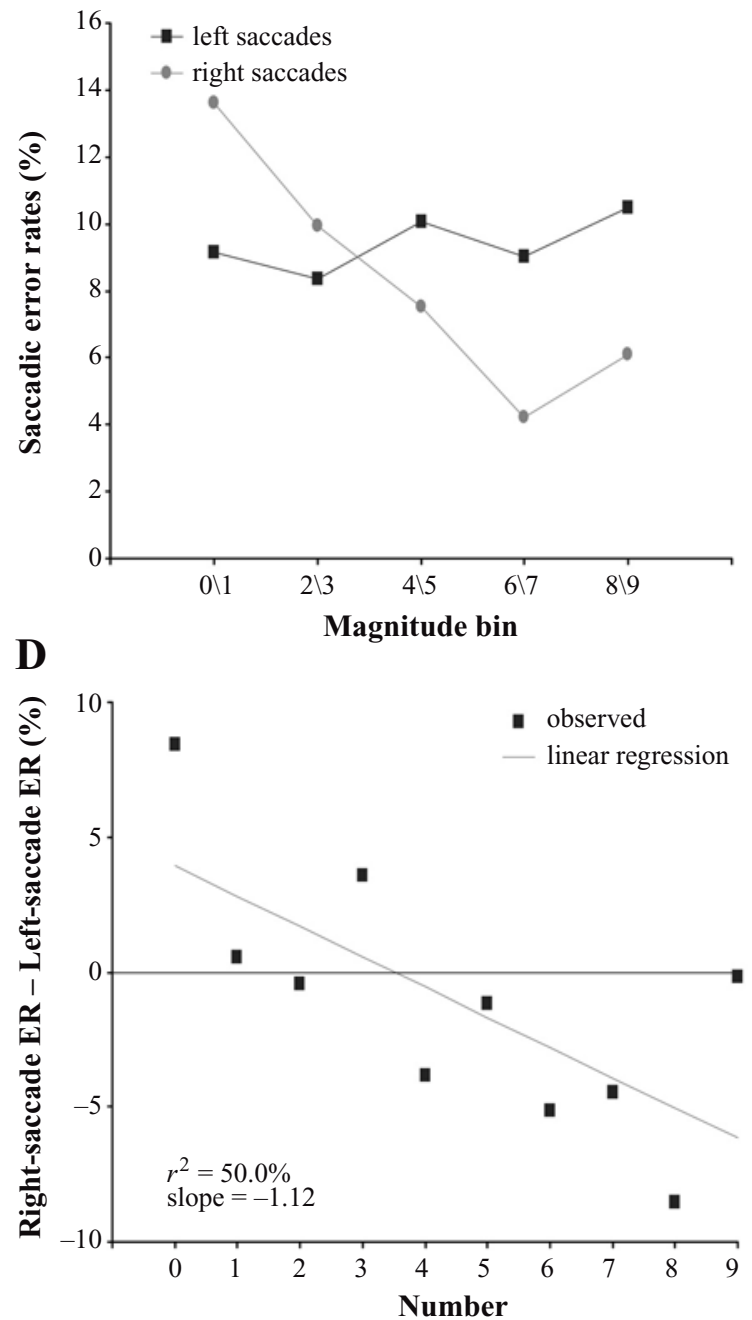

Figure 2. (A) Mean saccadic onset latencies (in milliseconds) in the saccadic response condition in Experiment 1 for saccades to the left (squares) and saccades to the right (dots) as a function of numerical magnitude. (B) Error rates (in percentages) in the saccadic response condition in Experiment 1 for saccades to the left (squares) and saccades to the right (dots) as a function of numerical magnitude. (C) Difference in saccadic latencies (SLs, in milliseconds) between saccades to the right and saccades to the left as a function of number $(0,1,2, \ldots, 9)$ in the saccadic response condition in Experiment 1. Solid line: regression of SL difference on number. (D) Difference in error rates (ERs, in percentages) between saccades to the right and saccades to the left as a function of number $(0,1,2, \ldots, 9)$ in the saccadic response condition in Experiment 1. Solid line: regression of ER difference on number. 
time. Second, we found a strong interaction between response side and magnitude [SNARC effect; $F(4,60)=$ $\left.4.40, M S_{\mathrm{e}}=898.93, p<.005\right]$. Figure $2 \mathrm{~A}$ illustrates that the latency difference between saccades to the right minus saccades to the left decreased monotonically with numerical magnitude. This saccadic SNARC effect occurred in a similar way for odd and even digits separately $[F(4,60)=$ $1.00, p>.40$, for the nonsignificant triple interaction of response side, magnitude, and parity].

A regression analysis of the type done with manual RTs yielded a mean regression slope equal to $-5.2[t(15)=$ $-2.45, S E M=2.13, p<.03]$. Thus, on average, the onset latency difference between saccades to the right versus saccades to the left decreased by $5.2 \mathrm{msec}$ per digit.

Error rates. The overall error rate was $8.8 \%$. Error rate decreased with magnitude $\left[F(4,60)=4.82, M S_{\mathrm{e}}=\right.$ $0.013, p<.005]$, an effect that was due mainly to the odd digits $\left[F(4,60)=4.93, M S_{\mathrm{e}}=0.019, p<.005\right.$, for the interaction of parity and magnitude]. As with manual responses, there was again a clear SNARC effect (shown in Figure 2B) on saccadic error rates: With increasing numerical magnitude, error rates decreased for saccades to the right but tended to increase for saccades to the left $\left[F(4,60)=5.40, M S_{\mathrm{e}}=0.021, p<.001\right]$. This SNARC effect on saccadic error rates was stronger for odd than for even digits, resulting in a significant three-way interaction of response side, magnitude, and parity $[F(4,60)=$ $\left.3.08, M S_{\mathrm{e}}=0.018, p<.05\right]$.

\section{Comparisons Across Response Conditions}

In a final analysis, we compared the observed effects across the two response conditions, adding effector as a further within-subjects factor. The only effect of this factor on the latency data was an interaction with response side $\left[F(1,15)=5.05, M S_{\mathrm{e}}=829.9, p<.05\right]$ : Saccades to the right were $11 \mathrm{msec}$ faster than those to the left, and this right-side advantage was twice as large $(22 \mathrm{msec})$ for manual responses. More central to our hypotheses, the SNARC effects on RT and SL reported above were of similar size and direction in both the manual and the saccadic response conditions $[F(4,60)=0.09, p>.98$, for the triple interaction of response side, magnitude, and effector]. The same result was obtained when the slope values from the regression analyses of manual latencies and SLs were compared $[t(15)=0.40, S E M=1.56, p>.70]$.

Similar results hold for error rates. The only effect of effector was that overall, with saccadic responses, more errors were observed than with manual responses $[F(1,15)=$ $\left.7.57, M S_{\mathrm{e}}=0.201, p<.02\right]$, perhaps because no immediate error feedback was given in the saccadic response condition. Again, the SNARC error rate effect occurred in very similar ways under both response conditions $[F(4,60)=0.74, p>.55$, for the triple interaction of response side, magnitude, and effector].

\section{Discussion}

The results of Experiment 1 are readily summarized. First, when the participants indicated the parity of digits manually, a systematic relation was found between the (in principle, irrelevant) numerical magnitude of the digit and the relative speed of left-hand responses versus right-hand responses. Specifically, the difference of righthand RT minus left-hand RT decreased with increasing numerical magnitude (manual SNARC effect; Dehaene et al., 1993). The SNARC effect showed up very clearly for error rates as well, so that the latency results cannot be attributed to simple speed-accuracy tradeoff mechanisms. Also, the manual SNARC effect occurred for even and odd digits separately in essentially the same manner.

Second, when the participants indicated the parity of a digit by horizontal saccades, the difference between onset latencies of saccades to the right and saccades to the left also decreased with numerical magnitude (saccadic SNARC effect): Eye movements to the left started earlier with smaller than with larger numbers, whereas eye movements to the right started earlier with larger than with smaller numbers. This effect was also accompanied by a pattern of error rates that increased with numerical magnitude for saccades to the left and decreased with numerical magnitude for saccades to the right.

Third, comparisons across the manual versus the saccadic response conditions indicated that the SNARC effect on both latency and error data occurred qualitatively and quantitatively in very similar ways under both the manual and the saccadic response conditions. For example, even the idiosyncratic finding that the parity of 7 was consistently easier to judge than that of 6 (whereas for all the other magnitude bins, even and odd judgments did not differ) occurred nearly identically in the manual and the saccadic latency and error data.

Taken together, these results constitute strong direct evidence against the manual association hypothesis as an explanation of the SNARC effect. Rather, they support the view that in accessing parity information, a magnitude representation is activated as well and that this representation of numerical magnitude is inherently space related, independently of the specific effector system onto which the overt response is to be mapped.

These conclusions are also supported by complementary evidence from analyses of the spatial characteristics of the actual eye movements. Specifically, if motor programming and execution processes played an important role in generating or modulating the saccadic SNARC effect, one might expect that the effect should also be reflected in the kinematic characteristics of the executed movements. For example, with the digit 9, the amplitudes of saccades to the right (i.e., in the odd-right condition) might be larger than the corresponding saccadic amplitudes to the left in the odd-left condition. However, the data did not give the slightest indication of any systematic magnitude-related effect on saccadic amplitudes; a similar conclusion holds with respect to the duration of the saccades as well. Thus, saccades to the left (to the right) are initiated earlier with numerically small (numerically large) digits, presumably because preceding representational processing stages are completed earlier, 
but the temporal and spatial characteristics of the actual eye movements themselves do not exhibit magnituderelated effects. This, too, complements and extends the analogous finding, mentioned in the introduction, that with manual responses the separation of the response buttons does not seem to modulate the size of the SNARC effect.

Given the evidence from Experiment 1 that the SNARC effect indeed reflects an early, effector-independent representational processing stage, the saccadic response condition can be exploited to further explore the internal topology of magnitude representations. As was discussed in the introduction, Dehaene (1997, pp. 82-83) has put forth the conjecture that there might be a minor vertical axis to our mental number representation, in the sense that smaller versus larger numerical magnitudes might be preferentially represented as bottom versus top, respectively. In view of the natural left-/right-hand dichotomy, it is difficult to test this conjecture with manual responses. Therefore, our Experiment 2 had two aims. First, we wanted to test whether saccadic responses in a parity task would show a vertical SNARC effect. Second, if this effect does exist, we wanted to determine whether it would occur in ways functionally similar to the horizontal saccadic SNARC effect observed in Experiment 1.

\section{EXPERIMENT 2}

Experiment 2 tested for a vertical saccadic SNARC effect by comparing the latencies of upward versus downward saccades in a parity task involving numbers of varying magnitude.

\section{Method}

\section{Participants}

Sixteen (12 female) right-handed students at the University of Nijmegen, 18-42 years of age, participated in two separate sessions in return for 15 Euro $(\approx \$ 15)$.

\section{Stimuli and Apparatus}

The stimuli and apparatus were the same as those in the saccadic response condition in Experiment 1, except that the left and right response boxes containing the target dot for the saccades were now replaced with a lower and an upper response box placed $8^{\circ}$ below and above the fixation cross.

\section{Procedure}

The experiment consisted of two sessions on separate days, one for each mapping of parity to response location (even-down/odd-up vs. even-up/odd-down); the order of the mapping was counterbalanced across participants. The task was to indicate the parity of the number presented by a vertical saccade. In the even-down mapping, the participants were instructed to saccade to the center dot in the lower box when the number was even and to the center dot in the upper box when the number was odd. In the even-up condition, the parity-to-response mapping was reversed. All other procedural aspects were identical to those for the saccadic response condition in Experiment 1. Experiment 2 contained no manual response condition. Each complete session took approximately $90 \mathrm{~min}$.

\section{Preliminary Data Reduction}

A valid trial had to meet the following five criteria: (1) The preceding green central cross was properly fixated during the last $200 \mathrm{msec}$ before the digit appeared, (2) the saccade started between
200 and 1,200 msec after digit onset, (3) the vertical saccade amplitude was between $5^{\circ}$ and $11^{\circ}$, (4) the horizontal saccade amplitude was smaller than $3^{\circ}$, and (5) the saccade duration was between 20 and $120 \mathrm{msec}$. Across all participants, $26.1 \%$ of the trials did not pass one or more of these criteria; these invalid trials were excluded from all further analyses.

Mean SLs and error rates were subjected to repeated measures $2 \times$ $2 \times 5$ ANOVAs, with factors of parity (2: even vs. odd), direction of saccade (2: downward vs. upward), and numerical magnitude ( 5 bins: $0 / 1,2 / 3,4 / 5,6 / 7$, and $8 / 9$ ). Comparisons across the saccadic response conditions in Experiments 1 versus 2 were based on four-way repeated measures ANOVAs, with the additional between-subjects factor of orientation of response axis (2: horizontal vs. vertical).

\section{Results}

\section{Saccadic Latency}

None of the three factors of magnitude, saccade direction, and parity had a significant main effect on SL. Figure $3 \mathrm{~A}$ illustrates that the onset latency of saccades directed to the bottom location increased with numerical magnitude, whereas the onset latency of saccades directed to the top location decreased with numerical magnitude. Thus, we obtained a clear vertical SNARC effect-namely, a monotone decrease of the difference between SL for upward saccades minus SL for downward saccades as a function of numerical magnitude $\left[F(4,60)=2.99, M S_{\mathrm{e}}=\right.$ $545.58, p<.05]$. When this vertical SNARC effect was considered separately for odd and even digits, its size was larger for even than for odd digits, yielding a triple interaction of saccade direction, magnitude, and parity $\left[F(4,60)=4.10, M S_{\mathrm{e}}=332.63, p<.01\right]$. As in Experiment 1 , odd and even judgments in each magnitude bin were usually equally fast, except again for the magnitude bin containing the digits 6 (which was the slowest digit overall; $421 \mathrm{msec}$ ) and 7 (the fastest digit overall; $388 \mathrm{msec}$ ), yielding a significant magnitude $\times$ parity interaction $\left[F(4,60)=7.49, M S_{\mathrm{e}}=646.80, p<.001\right]$.

A regression analysis of the SLs yielded a mean regression slope equal to $-3.0[t(15)=-3.06, S E M=0.98$, $p<.01]$. Thus, on average, the onset latency difference between upward saccades and downward saccades decreased by $3.0 \mathrm{msec}$ per digit.

\section{Error rates}

The overall error rate was $8.2 \%$. None of the factors had a main effect on error rate, but the interaction of magnitude with saccade direction was significant $[F(4,60)=$ $\left.3.96, M S_{\mathrm{e}}=0.019, p<.01\right]$. This vertical SNARC effect on error rates is shown in Figure 3B, and it occurred similarly when odd and even digits were considered separately $\left[F(4,60)=0.74, M S_{\mathrm{e}}=0.012, p>.55\right.$, for the interaction of saccade direction, magnitude, and parity]. The only other significant effect was an interaction of magnitude with parity $\left[F(4,60)=3.60, M S_{\mathrm{e}}=0.021, p<\right.$ $.02]$, reflecting mainly a lower error rate for 7 than for 6 .

\section{Comparing the Vertical and the Horizontal Saccadic SNARC Effect}

In a final analysis, we compared the vertical saccadic SNARC effect as observed in the present experiment with the horizontal saccadic SNARC effect observed in 
A

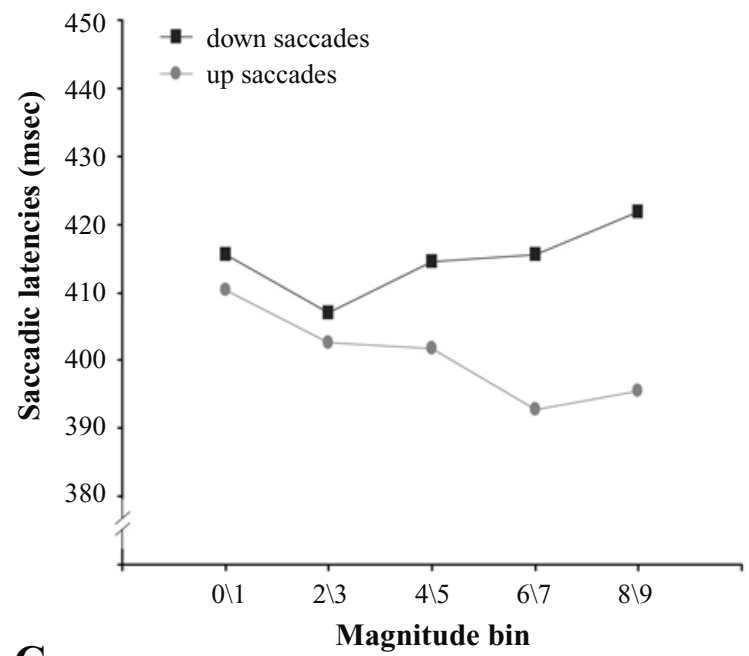

C

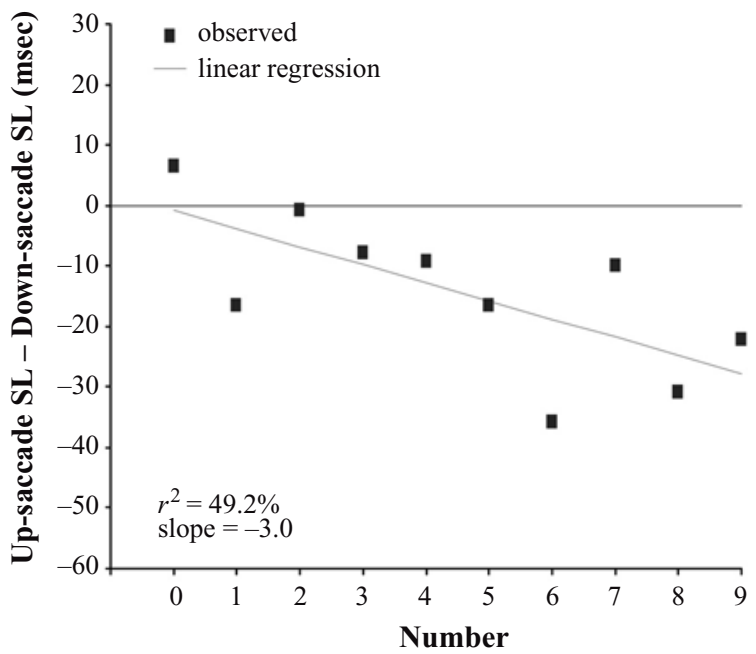

B

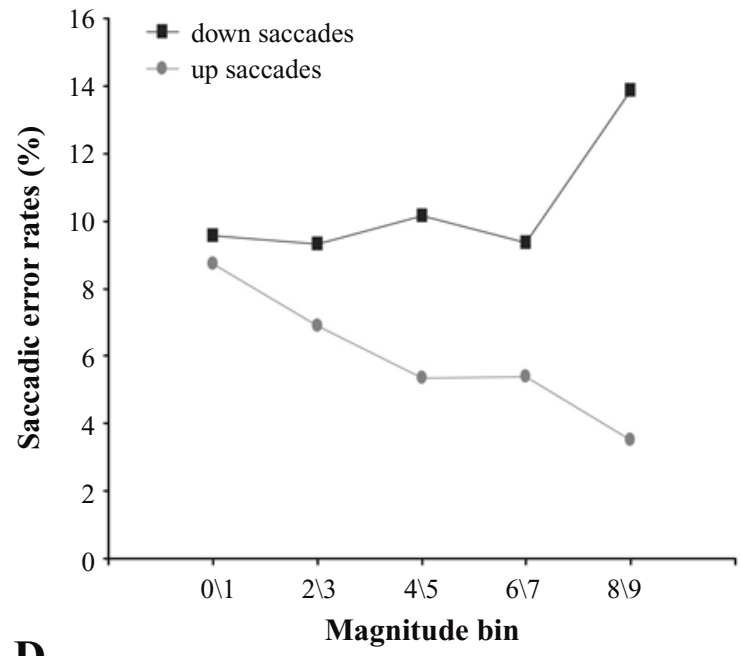

D

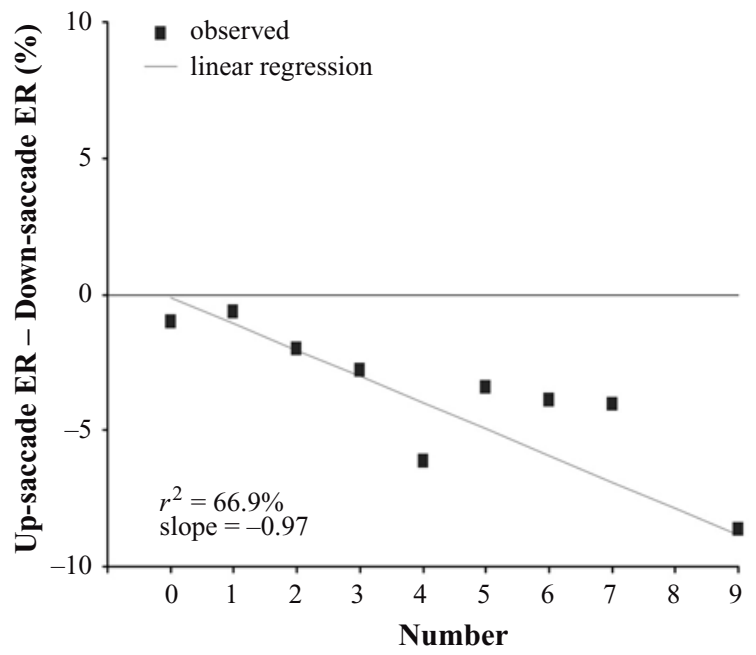

Figure 3. (A) Mean saccadic onset latencies ( in milliseconds) in Experiment 2 for saccades to the bottom (squares) and saccades to the top (dots) as a function of numerical magnitude. (B) Error rates (in percentages) in Experiment 2 for saccades to the bottom (squares) and saccades to the top (dots) as a function of numerical magnitude. (C) Difference in saccadic latencies (SLs, in milliseconds) between saccades to the top and saccades to the bottom as a function of number $(0,1,2, \ldots, 9)$ in Experiment 2. Solid line: regression of SL difference on number. (D) Difference in error rates (ERs, in percentages) between saccades to the top and saccades to the bottom as a function of number $(0,1,2, \ldots, 9)$ in Experiment 2. Solid line: regression of ER difference on number.

Experiment 1 by adding the orientation of the response axis (horizontal vs. vertical) as a between-subjects factor. The only significant effect of this factor on saccadic latency was a four-way interaction: The modulation of the SNARC effect by parity (i.e., that the magnitude $X$ saccade direction interaction tended to be larger with even digits) was stronger for vertical than for horizontal saccades $\left[F(4,120)=2.64, M S_{\mathrm{e}}=421.4, p<.05\right]$. All other main effects or interactions held in a similar way for both orientations of the response axis; in particular, the SNARC effect (i.e., the magnitude $\times$ saccade direction interaction) occurred similarly for horizontal as well as vertical saccades $\left[F(4,120)=0.52, M S_{\mathrm{e}}=722.4, p>.70\right]$.

These conclusions are further supported when we compare the slope results from the regression analyses of the horizontal versus vertical saccadic SNARC effects. Under both conditions, the onset latency difference of saccades to the right versus the left or to the top versus the bottom decreased systematically with numerical magnitude, but the difference between the slopes for horizontal versus vertical saccades was not significant $[t(30)=0.94, S E M=$ $2.34, p>.35]$. 
A corresponding across-experiments comparison of error rates yielded no significant main effect or interaction involving the factor of orientation of the response axis.

\section{Discussion}

The results of Experiment 2 demonstrate, first, that there is a clear association of numerical magnitude with spatial response codes along the vertical meridian. Specifically, when participants indicate the parity of a digit by vertical saccades, the latency difference between upward saccades and downward saccades decreases with numerical magnitude (vertical SNARC effect): Eye movements to the lower response location start earlier with smaller than with larger numbers, whereas eye movements to the upper response location start earlier with larger than with smaller numbers. This effect is paralleled by an analogous pattern of error rates that increase with numerical magnitude for saccades to the bottom and decrease with numerical magnitude for saccades to the top. The size of the SNARC effects on both saccadic latency and response accuracy for vertical saccades is similar to that found for horizontal saccades in Experiment 1. These results are consistent with the view that numerical magnitude has a two-dimensional internal representation, much like an internal number map. Alternatively, our results are also consistent with the assumption of two functionally independent number representations or even with a single representation that can be adaptively reoriented according to the specific task demands.

Second, the present results again confirm the interpretation that the SNARC effect is not dependent on, or limited to, overlearned manual motor associations but reflects a space-related internal representation of numerical magnitude. Our results suggest that the organization of this internal representation is not strictly unidimensional-for example, like a mental number line (Restle, 1970). A more general concept by which to describe this organization might be a number map or field, the lower left quadrant of which is preferentially associated with smaller numbers, whereas larger numbers are associated with the right upper quadrant. In this view, the standard horizontal SNARC effect could reflect the projections of a two-dimensional representation onto the horizontal axis. The broader implications of these findings will be discussed in the next section.

\section{GENERAL DISCUSSION}

Dehaene et al. (1993) first demonstrated that when the parity status of digits has to be judged in a bimanual response task, left-hand responses are faster with small than with large numbers, whereas right-hand responses are faster with large than with small numbers (SNARC effect). As was reviewed in the introduction, the SNARC effect holds across a range of different conditions, number notations, and tasks. According to one view, it reflects a genuine left-to-right orientation of our internal number representation, often likened to the concept of a mental number line (e.g., Butterworth, 1999; Dehaene et al., 1993; Restle, 1970). If so, the effect should presumably occur in a similar way with any effector that is capable of distinct left-right orientations. An alternative view is based on the observation that many effects are surprisingly effector specific; for example, Milner and Goodale (1995) have demonstrated how the haptic and visual grasping response can be systematically dissociated. On this view, the SNARC effect originates with overlearned motor associations between particular numbers and particular manual responses. Such lateralized motor associations might have been formed, for example, by our life-long experience with manually operated number-related devices, such as typewriters or keyboards, and they may have evolved quite independently of how these numbers are actually internally represented; in fact, superficially similar manual associations and preferences occur within other, strictly nonnumerical domains as well (e.g., in handling cutlery).

All studies in which the SNARC effect has been explored so far have relied on manual responses; therefore, it is difficult to know from extant data to what degree the effect depends on specific manual associations, although, as was reviewed in the introduction, part of the existing evidence seems more in line with the number line interpretation. The present study addressed this issue by using a saccadic response situation and by comparing it with the standard bimanual response condition.

The results of Experiment 1 yielded two new major findings. First, a clear SNARC effect was observed for saccadic onset latency and for saccadic error rate as well; this is a direct demonstration that the effect is not limited to experimental conditions in which the participants indicate their parity decisions bimanually. Second, this saccadic SNARC effect had the same size and showed similar characteristics as the standard manual SNARC effect. Clearly, these findings are further and direct support for the view that the SNARC effect originates with a spacerelated central magnitude representation and is then passed on and propagated to later processing stages, such as response preparation and execution. On the basis of this conclusion, Experiment 2, in which a saccadic response condition was again used, addressed the open question of whether a corresponding SNARC effect exists for the vertical orientation as well. The two most important findings were, first, that this vertical SNARC effect does indeed exist when upward and downward saccades to the same digits are compared and second, that the size and characteristics of this effect are quite comparable to those found for horizontal saccades in Experiment 1.

Taken together, both experiments provide strong support for the view that the SNARC effect is of central representational origin and manifests itself in similar ways with different effectors. In fact, recent findings of Fischer et al. (2003) suggested that an automatic space-related representation may even facilitate covert shifts of visual 
attention in a detection task with no antagonistic manual responses. This magnitude representation is likely to be richer than a one-dimensional number line and could be likened to an internal number map, although two independent representations, or a single representation with an adaptive task-dependent orientation, are not ruled out by Experiment 2 either. These conclusions immediately suggest several relevant and testable predictions for further experiments.

First, if the functional locus of the SNARC effect is indeed at a relatively early, representational stage of processing, it should in principle be possible to observe its neural correlates, with temporal onsets that precede the preparation and execution of the overt manual response. More specifically, it should then be possible to find a significant event-related potential (ERP) signature of the response side $\times$ magnitude interaction that precedes the start of later processing stages such as response activation, as indicated by the onset of the lateralized readiness potential (for details of this ERP-based chronometric logic, see Dehaene, 1996; Otten et al., 1996; Schwarz \& Heinze, 1998).

Second, if, indeed, effects of different response mappings are only a manifestation of the SNARC effect but its origin is at a central representational level, it should in principle be possible to obtain the effect even without varying and comparing the assignment of lateralized responses (cf. the study of Fischer et al., 2003, described above). For example, SNARC-like effects should then arise with lateralized presentations of the digits - even if the responses (e.g., verbal) required of the participants are not lateralized. Specifically, small numbers presented to the right and large numbers presented to the left should then constitute a presentation format that is more incompatible with our internal representation of number than is the converse mapping (cf. Tlauka, 2002).

Third, and related to the previous prediction, if the comparison of different hand mappings per se is (convenient but) not critical to the SNARC effect, it should presumably also be found by varying the response mappings within a single hand. For example, if we were to vary the way in which odd and even are mapped onto the index and middle fingers of the dominant hand, we would still expect a SNARC effect if this effect actually originates at more central representational stages.

Fourth, if, as Experiment 2 suggests, numbers are internally represented in a two-dimensional map-like structure with a genuine bottom-left to top-right magnitude orientation, we also would expect a strong SNARC effect for a diagonal saccadic response condition in which eye movements are either to a lower left or an upper right response location. Indeed, if the separate SNARC effects for each orientation sum or coactivate, this diagonal saccadic SNARC effect would be predicted to be larger than that for either the horizontal or the vertical dimension alone. Conversely, we would by the same reasoning expect a cancellation or, at least, a degradation of the sep- arate SNARC effects in each dimension along the negative diagonal from upper left to lower right saccadic response locations.

A final conclusion concerns the question of which tasks we expect to yield a SNARC effect. By far, the greatest number of studies of the SNARC effect have used parity judgments, a task that is known to be relatively slow when compared with magnitude judgments (e.g., Otten et al., 1996; Sudevan \& Taylor, 1987). Also, the parity status of a number is logically independent of its magnitude, but it is still an inherently number-related property; under most experimental conditions, accessing and retrieving parity information seems difficult or impossible without activating in parallel magnitude-related information about the number presented (Bächtold et al., 1998; Berch et al., 1999; Butterworth, 1999; Dehaene et al., 1993). If the SNARC effect arises at a relatively early stage of processing, during which magnitude-related information is automatically activated, we would expect it to show up even with tasks of (1) a much simpler, nonnumerical nature and (2) a correspondingly faster time course. Fias et al. (1996) addressed the first issue by using a phoneme monitoring task in which participants judged whether the phoneme /e/ occurred in the name of a visually presented digit. Despite the nonnumerical nature of this task, they obtained a clear SNARC effect, indicative of a fast and automatic activation of semantic, magnitude-related information, in line with the present conclusions. If this interpretation is valid, the SNARC effect should presumably not be limited to this relatively slow nonnumerical task (which is again about $100 \mathrm{msec}$ slower than parity judgments) but should also occur when more accidental nonnumerical features of digits are to be judged.

In addition, a disadvantage of both the parity and the phoneme tasks in terms of experimental design is that for every given digit, its parity status or phoneme content is a fixed property that cannot be experimentally varied. It is precisely this design feature that necessitates that, across blocks or sessions, the parity- or phoneme-toresponse mapping must be varied by different response instructions. Consider, however, a task in which a completely accidental digit attribute, such as its color (e.g., red/green), must be judged (Fias, Lauwereyns, \& Lammertyn, 2001; Lammertyn, Fias, \& Lauwereyns, 2002). This task does not require the comparison of different response mappings run in separate blocks, because both left and right (or upward and downward) responses to the same number are naturally obtained within a single block consisting of a random sequence of colored digits. If the SNARC effect originates with an early, automatic activation of a magnitude representation, we would predict a SNARC effect for purely accidental digit features as well, a prediction that Fias et al. (2001) have been able to confirm for some (e.g., orientation), but not for other (e.g., color), features. Fias et al. (2001) and Lammertyn et al. (2002) have attributed this dissociation to the different amounts by which these features activate the pari- 
etal cortex and, thus, overlap with number processing. Similarly, Pansky and Algom (2002, note 7) have recently reported the absence of number-space associations for judgments of the numerosity of digits, indicating that with this particular task, which is relatively elementary and fast, there is no evidence for an automatic activation of numerical magnitude.

In conclusion, the results of the present experiments provide new and direct evidence that the number-space associations consistently found in bimanual parity judgment tasks cannot be attributed to preferred and overlearned manual motor associations. Rather, the SNARC effect manifests itself in essentially similar ways with other effectors that are normally less (or not) systematically associated with small versus large numbers than are hands. Our findings support the interpretation that in parity judgment tasks, space-related magnitude representations are automatically activated and modulate the efficiency with which a horizontal or a vertical motor response is given. The present results also extend our view on the nature of this magnitude representation: They suggest that it is richer than a strictly unidimensional code, such as a number line, and is better characterized as a number map. More work will be required to further test the various and detailed predictions, described above, implied by our conclusions, so as to better understand how, why, and when our representations of number and space are closely interlocked.

\section{REFERENCES}

Algom, D., Dekel, A., \& Pansky, A. (1996). The perception of number from the separability of the stimulus: The Stroop effect revisited. Memory \& Cognition, 24, 557-572.

AsHCRAFT, M. H. (1992). Cognitive arithmetic: A review of data and theory. Cognition, 44, 75-106.

B ÄChtold, D., Baumüller, M., \& Brugger, P. (1998). Stimulusresponse compatibility in representational space. Neuropsychologia, 36, 731-735.

BERCH, D. B., Foley, E. J., Hill, R. J., \& RYAN, P. M. (1999). Extracting parity and magnitude from Arabic numerals: Developmental changes in number processing and mental representation. Journal of Experimental Child Psychology, 74, 286-308.

BeSNer, D., \& Coltheart, M. (1979). Ideographic and alphabetic processing in skilled reading of English. Neuropsychologia, 17, 467-472.

Bishop, Y. M. M., FienberG, S. E., \& Holland, P. W. (1975). Discrete multivariate analysis. Cambridge, MA: MIT Press.

ButTERWORTH, B. (1999). The mathematical brain. London: Macmillan.

Chochon, F., Cohen, L., van de Moortele, P. F., \& Dehaene, S. (1999). Differential contributions of the left and right inferior parietal lobules to number processing. Journal of Cognitive Neuroscience, 11, 617-630.

Colby, C. L., \& Goldberg, M. E. (1999). Space and attention in parietal cortex. Annual Review of Neuroscience, 22, 319-349.

Corballis, M. C. (1994). Can commissurotomized subjects compare digits between the visual fields? Neuropsychologia, 32, 1475-1486.

DeHAEne, S. (1996). The organization of brain activations in number comparison: Event-related potentials and the additive-factors method. Journal of Cognitive Neuroscience, 8, 47-68.

DeHAENE, S. (1997). The number sense: How the mind creates mathematics. New York: Oxford University Press.

Dehaene, S., \& Akhavein, R. (1995). Attention, automaticity, and levels of representation in number processing. Journal of Experimental Psychology: Learning, Memory, \& Cognition, 21, 314-326.
Dehaene, S., Bossini, S., \& Giraux, P. (1993). The mental representation of parity and numerical magnitude. Journal of Experimental Psychology: General, 122, 371-396.

Dehaene, S., Dehaene-Lambertz, G., \& Cohen, L. (1998). Abstract representations of numbers in the animal and the human brain. Trends in Neurosciences, 21, 355-361.

Dehaene, S., Spel ke, E., Pinel, P., Stanescu, R., \& Tsivkin, S. (1999). Sources of mathematical thinking: Behavioral and brainimaging evidence. Science, 284, 970-974.

FIAS, W. (2001). Two routes for the processing of verbal numbers: Evidence from the SNARC effect. Psychological Research, 65, 250-259.

Fias, W., Brysbaert, M., Geypens, F., \& D'Ydewalle, G. (1996). The importance of magnitude information in numerical processing: Evidence from the SNARC effect. Mathematical Cognition, 2, 95-110.

Fias, W., Lauwereyns, J., \& Lammertyn, J. (2001). Irrelevant digits affect feature-based attention depending on the overlap of neural circuits. Cognitive Brain Research, 12, 415-423.

FISCHER, M. H. (2001). Number processing induces spatial performance biases. Neurology, 57, 822-826.

Fischer, M. H., Castel, A. D., Dodd, M. D., \& Pratt J. (2003). Perceiving numbers causes spatial shifts of attention. Nature Neuroscience, 6, 555-556.

GALLISTEL, C. R. (2002). Language and spatial frames of reference in mind and brain. Trends in Cognitive Sciences, 6, 321-322.

Gallistel, C. R., \& Gelman, R. (1992). Preverbal and verbal counting and computation. Cognition, 44, 43-74.

Girelli, L., Lucangeli, D., \& ButTerworth, B. (2000). The development of automaticity in accessing number magnitude. Journal of Experimental Child Psychology, 76, 104-122.

GLOVER, S. R., \& DiXON, P. (2001). Dynamic illusion effects in a reaching task: Evidence for separate visual representations in the planning and control of reaching. Journal of Experimental Psychology: Human Perception \& Performance, 27, 560-572.

GLOVER, S. [R.], \& DiXON, P. (2002). Dynamic effects of the Ebbinghaus illusion in grasping: Support for a planning/control model of action. Perception \& Psychophysics, 64, 266-278.

Göbel, S., Walsh, V., \& Rushworth, M. F. S. (2001). The mental number line and the human angular gyrus. NeuroImage, 14, 1278-1289.

Lammertyn, J., Fias, W., \& Lauwereyns, J. (2002). Semantic influences on feature-based attention due to overlap of neural circuits. Cortex, 38, 878-882.

LATASH, M. L. (1999). Mirror writing: Learning, transfer, and implications for internal inverse models. Journal of Motor Behavior, $\underline{31}_{2}$ 107-111.

MCCLOSKEY, M. (1992). Cognitive mechanisms in numerical processing: Evidence from acquired dyscalculia. Cognition, 44, 107-157.

Milner, A. D., \& Goodale, M. A. (1995). The visual brain in action. London: Oxford University Press.

Otten, L. J., Sudevan, P., Logan, G. D., \& Coles, M. G. H. (1996). Magnitude versus parity in numerical judgements: Event-related brain potentials implicate response conflict as the source of interference. Acta Psychologica, 94, 21-40.

Pansky, A., \& Algom, D. (1999). Stroop and Garner effects in comparative judgment of numerals: The role of attention. Journal of Experimental Psychology: Human Perception \& Performance, 25, 39-58.

PANSKY, A., \& Algom, D. (2002). Comparative judgment of numerosity and numerical magnitude: Attention preempts automaticity. Journal of Experimental Psychology: Learning, Memory, \& Cognition, 28, 259-274.

RESTLE, F. (1970). Speed of adding and comparing numbers. Journal of Experimental Psychology, 83, 274-278.

RosenBaUM, D. A. (1991). Human motor control. San Diego: Academic Press.

Rubin, N., Nakayama, K., \& Shapley, R. (1996). Enhanced perception of illusory contours in the lower vs. upper visual hemifields. Science, 271, 651-653.

SCAMIDT, R. A. (1988). Motor control and learning: A behavioral emphasis (2nd ed.). Champaign, IL: Human Kinetics.

Schwarz, W., \& Heinze, H.-J. (1998). On the interaction of numerical 
and size comparison: A behavioral and event-related potential study. Neuropsychologia, 36, 1167-1179.

ScHWARZ, W., \& IscHEBECK, A. (2000). Sequential effects in number comparison. Journal of Experimental Psychology: Human Perception \& Performance, 26, 1606-1621.

ScHWARZ, W., \& ISCHEBECK, A. (2003). On the relative speed account of number-size interference effects in comparative judgments of numerals. Journal of Experimental Psychology: Human Perception \& Performance, 29, 507-522.

SchWARZ, W., \& STEIN, F. (1998). On the temporal dynamics of digit comparison processes. Journal of Experimental Psychology: Learning, Memory, \& Cognition, 24, 1275-1293.

SuDEVAN, P., \& TAYLOR, D. A. (1987). The cuing and priming of cognitive operations. Journal of Experimental Psychology: Human Perception \& Performance, 13, 89-103.
Tlauka, M. (2002). The processing of numbers in choice-reaction tasks. Australian Journal of Psychology, 54, 94-98.

Tzelgov, J., MeYer, J., \& HeNIK, A. (1992). Automatic and intentional processing of numerical information. Journal of Experimental Psychology: Learning, Memory, \& Cognition, 18, 166-179.

WAscher, E., Schatz, U., Kuder, T., \& VerLEger, R. (2001). Validity and boundary conditions of automatic response activation in the Simon task. Journal of Experimental Psychology: Human Perception \& Performance, 27, 731-751.

ZoRZI, M., Priftis, K., \& UMiLtà, C. (2002). Brain damage: Neglect disrupts the mental number line. Nature, 417, 138-139.

(Manuscript received April 3, 2003;

revision accepted for publication September 8, 2003.) 